\title{
TEMPO-mediated surface oxidation of cellulose whiskers
}

\author{
Youssef Habibi ${ }^{1,2}$, Henri Chanzy ${ }^{1}$ and Michel R. Vignon ${ }^{1, *}$ \\ ${ }^{1}$ Institut de Chimie Moléculaire de Grenoble (ICMG), Centre de Recherches sur les Macromolécules \\ Végétales (CERMAV-CNRS), Joseph Fourier University, BP 53, 38041 Grenoble cedex 9, France; ${ }^{2}$ Equipe \\ des Parois Végétales et Matériaux Fibreux, UMR FARE (INRA/URCA), CREA, 2 Espl. R. Garros, BP \\ 224, 51686 Reims cedex 2, France; *Author for correspondence (e-mail: Michel.Vignon@cermav.cnrs.fr; \\ phone: +33-4-76037614; fax: +33-4-76547203)
}

Received 25 March 2006; accepted in revised form 22 June 2006

Key words: Birefringent suspensions, Cellulose whiskers, TEMPO oxidation

\begin{abstract}
Cellulose whiskers resulting from $\mathrm{HCl}$ acid hydrolysis of tunicin were subjected to TEMPO-mediated oxidation under various conditions and the extent of the resulting oxidation was characterized by Fouriertransform infrared spectroscopy (FT-IR), conductimetry, X-Ray diffraction analysis and transmission electron microscopy (TEM). With degree of oxidation of up to 0.1 the samples kept their initial morphological integrity and native crystallinity, but at their surface the hydroxymethyl groups were selectively converted to carboxylic groups, thus imparting a negative surface charge to the whiskers. When dispersed in water these oxidized whiskers did not flocculate and their suspensions appeared birefringent when viewed between cross polarizers, thus indicating a liquid crystalline behavior.
\end{abstract}

\section{Introduction}

It has been known for a number of years that the hydrolysis of native cellulose with strong hydrochloric or sulfuric acid induced a rapid decrease of its degree of polymerization (DP) toward a lower value - the so called level-off DP (LODP) - that remained constant for quite a while, even during prolonged hydrolysis. The value of LODP has been shown to depend on the cellulose origin: typical values of 250 being recorded for hydrolyzed cotton (Battista 1950), 140-200 for bleached wood pulp (Battista et al. 1956) and up to 6000 for the highly crystalline Valonia cellulose (Kai 1976). During the hydrolysis, the nearly endless cellulose microfibrils become longitudinally cleaved into shorter crystalline objects, commonly called cellulose "whiskers". These whiskers have the same diameter as the initial microfibril, but lengths ranging from tenth of nanometer for samples from wood or cotton origin to several micrometers for tunicin or Valonia cellulose. In fact, there is a direct correspondence between the length of the cellulose whiskers and the LODP of the corresponding material, as it is generally accepted that these whiskers consist of fully extended cellulose chain segments well organized in perfect crystalline arrangement (Rånby 1951).

The description of the properties of the cellulose whiskers has been the focus of a number of studies, dealing with either their fundamental structural aspects (Sugiyama et al. 1991; Helbert et al. 1998) or their potential utilizations (Favier et al. 1995; De Souza Lima and Borsali 2004; Azizi Samir et al. 2005). In view of their very small sizes, these whiskers need to be handled as suspensions in aqueous or organic solvents, and for this, their dispersibilty in these mediums needs to be 
optimized. If the cellulose whiskers are prepared by hydrolysis in hydrochloric acid, their dispersibility is limited (Araki et al. 1998) and their aqueous suspension tends to flocculate. On the other hand, when sulfuric acid is used as hydrolyzing agent, it interacts also with the surface hydroxyl groups of cellulose to yield charged surface sulfate groups that promote a perfect dispersion of the whiskers in water (Revol et al. 1992; Dong et al. 1996). When these homogeneous suspensions are concentrated, they self-organize into spectacular liquid crystalline order (Revol et al. 1992), a feature similar to what occurs in non-flocculating suspensions of other rod-like particles, such as tobacco mosaic viruses (Oster 1950), DNA fragments (Livolant and Leforestier 1996) or poly(tetrafluoroethylene) whiskers (Folda et al. 1988).

One of the main disadvantages presented by the preparation of aqueous suspension of cellulose whiskers by the sulfuric acid method is that the resulting sulfates moieties at the whisker surfaces are rather labile, being in particular readily removed under mild alkaline conditions. The TEMPO-mediated oxidation is an alternate promising route to convert surface hydroxyl of cellulose into charged carboxyl entities that do not have the lability of sulfate groups. The use of this technique has been attracting a number of investigations since the first reports of De Nooy et al. (1994), which showed that only the hydroxymethyl groups of polysaccharides were oxidized, whereas the secondary hydroxyls remained unaffected. For cellulose, the TEMPO-mediated oxidation was applied to different cellulose samples, ranging from cotton linters, to wood pulp, parenchyma cell cellulose, rayon and cellulose III (Isogai and Kato 1998; Tahiri and Vignon 2000; Araki et al. 2001; da Silva Perez et al. 2003; Saito and Isogai 2004; Montanari et al. 2005; Saito et al. 2005, 2006). These studies have led to the preparation of a series of products, ranging from water-soluble polyuronic acid to partially derivatized cellulose products. In order to obtain a better control on the TEMPO-based chemical modification of cellulose with the goal of gaining fundamental information about the surface modification cellulose, we have chosen in this work to study the TEMPO-mediated oxidation of tunicin whiskers. These elements can be considered as models of crystalline cellulose and for them, we have searched oxidation conditions where the surface of the crystals would be selectively oxidized, while leaving intact the core of the crystals.

\section{Materials and methods}

\section{Preparation of cellulose whiskers}

Animal cellulose, i.e. tunicin, was extracted from the mantle of Halocynthia roretzi. Small fragments of the mantles were first treated with a solution of $\mathrm{KOH}(5 \% \mathrm{w} / \mathrm{v})$ overnight followed by washing and three successive bleaching treatments according to the method of Wise et al. (1946). The bleached fragments were disintegrated in water with a Waring Blendor. Cellulose whiskers were obtained by acid hydrolysis with $2.5 \mathrm{~N} \mathrm{HCl}$ solutions at reflux for $45 \mathrm{~min}$ and under continuous stirring. The resulting suspension was filtered and washed with water until neutrality, dialyzed against de-ionized water and then filtered and concentrated to constitute the stock suspension, which was used to prepare oxidized cellulose whiskers.

\section{TEMPO-mediated oxidation of cellulose whiskers}

About $510 \mathrm{mg}$, i.e. $3.15 \mathrm{mmol}$ of equivalent anydroglucose unit (AGU) of cellulose whiskers were suspended in water $(100 \mathrm{ml})$ and sonicated with a Branson Sonifier for $5 \mathrm{~min}$. TEMPO (14.75 mg, $0.094 \mathrm{mmol}$ ) and $\mathrm{NaBr}$ (162 mg, $1.57 \mathrm{mmol}$ ) were added to the suspension. A certain amount of the $1.24 \mathrm{M} \mathrm{NaOCl}$ solution, corresponding to $0.06-0.5$ molar ratio of $\mathrm{NaOCl} / \mathrm{AGU}$, was added slowly to the cellulose suspension. The $\mathrm{pH}$ of the mixture was maintained to 10 at room temperature by adding $0.5 \mathrm{M} \mathrm{NaOH}$ while stirring the suspension. After times ranging from 30 to $45 \mathrm{~min}$, the oxidation was terminated by adding methanol (ca. $1 \mathrm{ml}$ ) and the $\mathrm{pH}$ was adjusted to 7 with $0.5 \mathrm{M} \mathrm{HCl}$. The water insoluble fraction was recovered by centrifugation and washed thoroughly with water. The oxidized cellulose whiskers were dialyzed against distilled water and then freezedried and weighed to measure the mass recovery ratios.

\section{$X$-ray diffraction analysis}

X-ray measurements were made on pellets of cellulose whiskers. The X-ray diagrams were 
recorded on a Warhus flat film vacuum X-ray camera mounted on a Philips PW 1720 X-ray generator operated at $30 \mathrm{kV}$ and $20 \mathrm{~mA}$ with $\mathrm{Ni}$ filtered $\mathrm{CuK} \alpha$ radiation. Calibration was achieved with calcite. The degree of crystallinity of the samples was calculated, using the method of Segal et al. (1959).

\section{Transmission electron microscopy}

Drops of a cellulose whiskers suspension in water $(0.01 \% \mathrm{w} / \mathrm{v})$ were deposited on carbon-coated electron microscope grids and negatively stained with uranyl acetate and allowed to dry. The grids were observed with a Philips CM200 CRYO TEM operated at an accelerating voltage of $80 \mathrm{kV}$.

\section{Conductimetry}

The carboxyl content of oxidized cellulose samples was determined by conductimetric titration. The cellulose samples $(50 \mathrm{mg})$ were suspended into $15 \mathrm{ml}$ of $0.01 \mathrm{M}$ hydrochloric acid solutions. After 10 min of stirring, the suspensions were titrated with $0.01 \mathrm{M} \mathrm{NaOH}$. Typical titration curves are shown in Figure 1. These curves, which present a remarkable reproducibility, allow us to calculate the amount carboxyl groups and therefore the degree of oxidation (DO), which is given by the following equation (da Silva Perez et al. 2003),

$$
\mathrm{DO}=\frac{162 \times C \times\left(V_{2}-V_{1}\right)}{w-36 \times C \times\left(V_{2}-V_{1}\right)}
$$

where $C$ is the $\mathrm{NaOH}$ concentration $(\mathrm{mol} / \mathrm{l}), V_{1}$ and $V_{2}$ are the amount of $\mathrm{NaOH}$ (in 1$), w$ (g) the weight of the oven-dried sample; the value of 36 corresponds to the difference between the molecular weight of an AGU and that of the sodium salt of a glucuronic acid moiety.

\section{Infrared spectroscopy}

Infrared spectra were recorded on a FT-IR PerkinElmer 1720X spectrometer. Oxidized whiskers were converted to their acid form by ion exchange in order to displace the carboxyl absorption band toward higher wavelength and thus eliminate any interference with the absorbed water band

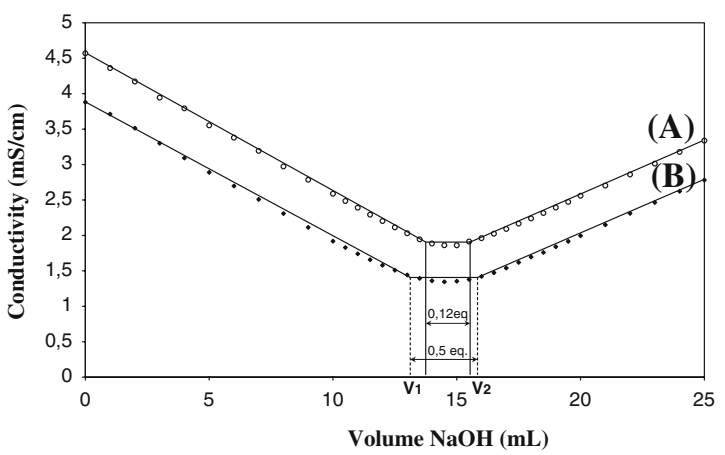

Figure 1. Conductimetric titration curves of oxidized cellulose whiskers resulting from different molar ratio of $\mathrm{NaOCl} / \mathrm{AGU}$. (A) 0.12 eq.; (B) 0.5 eq.

$\left(1640 \mathrm{~cm}^{-1}\right)$. These samples were then freeze-dried and analyzed as $\mathrm{KBr}$ pellets $(1 \%$ cellulose in anhydrous KBr). Spectra were recorded using a spectral width ranging from 400 to $4000 \mathrm{~cm}^{-1}$, a $2 \mathrm{~cm}^{-1}$ resolution, and an accumulation of 20 scans. DO measurements were achieved by calculating the ratio of the intensity of the absorption band near $1730 \mathrm{~cm}^{-1}$ - corresponding to the carbonyl stretching frequency - to that of the strongest band near $1050 \mathrm{~cm}^{-1}$, intrinsic of the cellulose backbone. Calibration was done using the conductimetric titration of the most oxidized specimen.

\section{Results and discussion}

\section{Chemical characterization of the oxidized cellulose} whiskers

The tunicin whiskers have the section of truncated lozenges (Helbert et al. 1998) that for simplification can be modeled as rectangles with average dimensions of long and short sides, respectively, $L=18.2 \mathrm{~nm}$ and $l=8.8 \mathrm{~nm}$ (Van Daele et al. 1992; Terech et al. 1999). In Figure 2, we show the representation of a model whisker and its orientation with respect to the section of the unit cell of cellulose $\mathrm{I} \beta$ and its two lattice parameters $a=0.801 \mathrm{~nm}$ and $b=0.817 \mathrm{~nm}$ (Sugiyama et al. 1991). In this model, the planes (110) corresponding to $0.61 \mathrm{~nm}$ are parallel to the long side of the rectangular whisker section whereas the $0.54 \mathrm{~nm}$ (110) are parallel to the short side. Thus within this average crystal, there are $(18.2 \times 8.8) /$ 


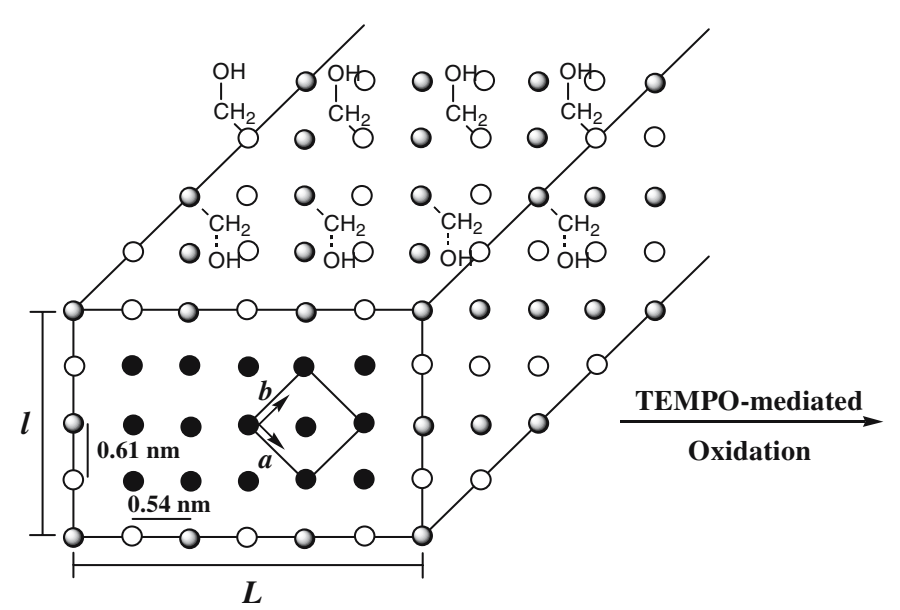

Inaccessible AGU at core crystal

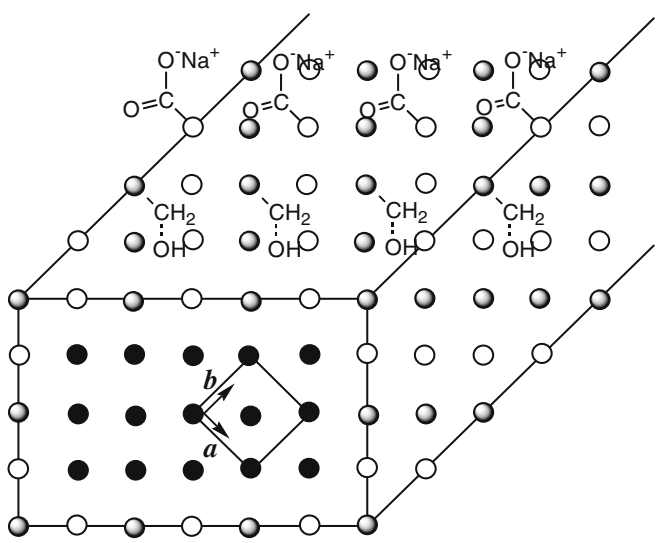

Accessible AGU at surface crystal

Figure 2. Schematic representation of a cellulose whisker cross-section.

$(0.61 \times 0.54)=486$ cellulose chains. At the surface, there are $2 \times(18.2 / 0.54)+2 \times(8.8 /$ $0.61)=96$ surface chains, which are susceptible to be oxidized. The ratio of surface chains to the total number of chains within the crystals is therefore of 0.19 . If we now consider the accessible hydroxymethyl groups, only half of these are available, the other being buried inside the crystalline whisker as a consequence of the two fold screw axis of the cellulose chain (Figure 2). Thus, the maximum degree of oxidation $\left(\mathrm{DO}_{\max }\right)$, that can be obtained for the average tunicin whiskers, is thus of $0.19 / 2$ $=0.095$, i.e of roughly 0.1 .

The properties and yields of the oxidized cellulose whiskers as a function of $\mathrm{NaOCl} / \mathrm{AGU}$ content are listed in Table 1. In this table, the DO measured by conductimetry increases with the amount of $\mathrm{NaOCl}$, to reach a plateau when the value of $0.2 \mathrm{NaOCl} / \mathrm{AGU}$ is reached. This value is significant as it corresponds to the stoichiometric surface oxidation of the whiskers, since the oxidation of one hydroxylmethyl group requires two
$\mathrm{NaOCl}$ molecules. Under this condition, the experiments indicate that the insoluble content of the sample is close to $100 \%$ whereas an experimental DO of 0.081 is obtained, a value very close to the $\mathrm{DO}_{\text {max }}$ value of 0.1 . Since, at this level of oxidation, only $5 \%$ of the sample has been solubilized, one can conclude that essentially all the surface accessible hydroxymethyl groups have been carboxylated and that conditions close to the stoichiometry have been reached. Quite interestingly and as expected, the use of an increased amount of $\mathrm{NaOCl}$ does not lead to a substantial increase either of the experimental DO or of the soluble fraction of the sample. The slight difference between the maximum experimental DO and $\mathrm{DO}_{\max }$ may be due to either to the experimental errors in the titration, or to the oversimplified cellulose model shown in Figure 2.

The oxidation of the whiskers can be detected in the FTIR spectra as shown in Figure 3. In these spectra, the arrowed band near $1730 \mathrm{~cm}^{-1}$ corresponds to the $\mathrm{C}=\mathrm{O}$ stretching frequency of

Table 1. Oxidized cellulose whiskers: yield, predicted and experimental degree of oxidation.

\begin{tabular}{lllllll}
\hline $\begin{array}{l}\text { Molar ratio of } \\
\text { NaOCl/AGU }\end{array}$ & Yield & & $\begin{array}{l}\text { Predicted } \\
\text { DO }\end{array}$ & Conductimetric & $\begin{array}{l}\text { FT-IR } \\
\text { DO }\end{array}$ & $\begin{array}{l}\text { Homogeneous } \\
\text { dispersion }\end{array}$ \\
\cline { 2 - 4 } & Soluble (\%) & Insoluble (\%) & & & \\
\hline 0.06 & 1 & 99 & 0.03 & 0.029 & 0.031 & no \\
0.12 & 2 & 98 & 0.06 & 0.056 & 0.059 & yes \\
0.2 & 5 & 95 & 0.1 & 0.081 & 0.082 & yes \\
0.5 & 6 & 94 & 0.1 & 0.097 & yes \\
\hline
\end{tabular}




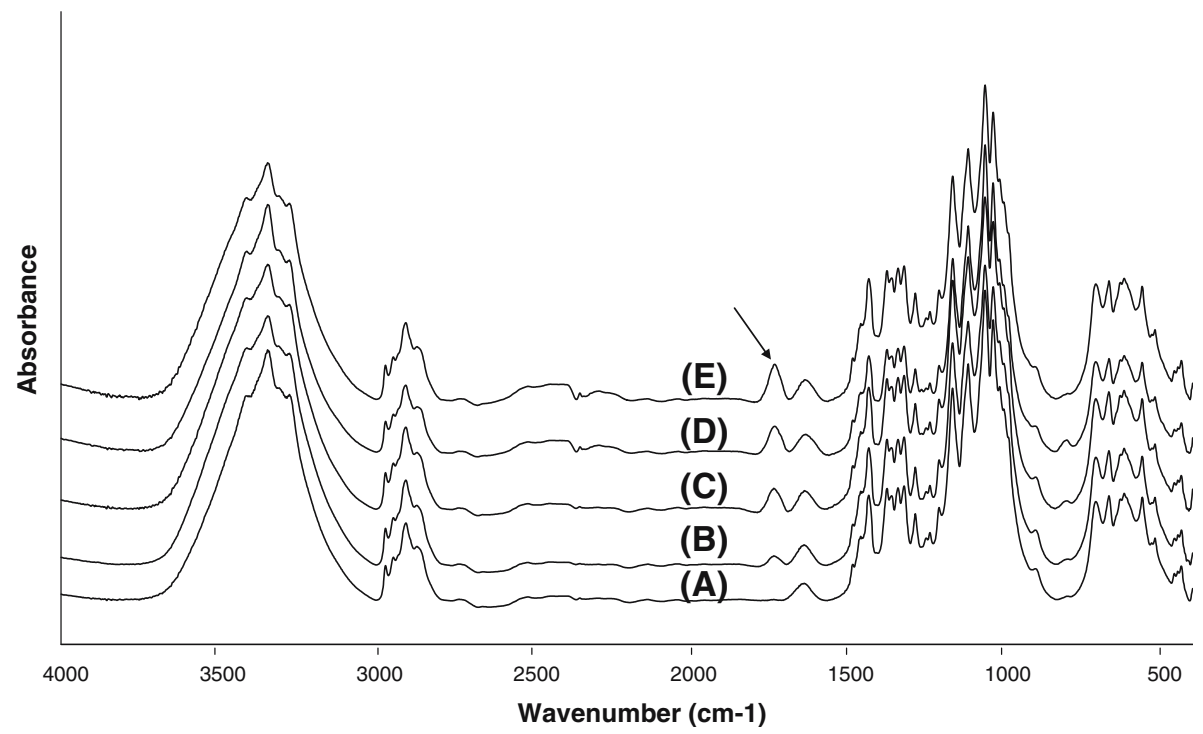

Figure 3. FT-IR spectrum of cellulose whiskers: (A) original non-oxidized; (B, C, D, E) oxidized with different molar ratio of $\mathrm{NaOCl} /$ AGU. (B) 0.06 eq.; (C) 0.12 eq.; (D) 0.2 eq.; (E) 0.5 eq.

carboxyl groups in their acidic form. The band is absent in the initial samples (spectrum 3A), but can be detected already in a sample where DO is only one third of $\mathrm{DO}_{\max }$ (spectrum $3 \mathrm{~B}$ ). A maximum value of this band is reached in the spectrum $3 \mathrm{E}$, corresponding to the maximum oxidation conditions that were applied here. The measurement of the intensity of this band and its comparison with the band near $1050 \mathrm{~cm}^{-1}$ allow us to confirm the variation of DO with respect of oxidation reagents (Table 1).

\section{Structural and morphological details of the oxidized} whiskers

Figure 4 illustrates a typical preparation of surface oxidized tunicin whiskers. This image is quite similar to those already published on sulfuric acid treated tunicin whiskers (Favier et al. 1995; Sassi and Chanzy 1995). As in these earlier preparations, Figure 4 indicates that there is a large polydispersity in the distribution of both the length and the width of the whiskers. Indeed, their length ranges from around $0.1 \mu \mathrm{m}$ for the shortest to more than $10 \mu \mathrm{m}$ for the longest ones, with an average size of $1 \mu \mathrm{m}$. Regarding their width, value of 5-20 nm are measured, but most of them are close to $20 \mathrm{~nm}$. This distribution of dimensions corresponds to those of the whiskers treated either with the sulfuric (Favier et al. 1995; Sassi and Chanzy 1995) or hydrochloric treatment. Thus, we can conclude that the TEMPO-mediated oxidation conditions that were used here do not have further hydrolytic treatment on the whiskers, whose lengths are determined by the initial hydrolytic treatment. Regarding the whisker width and their distribution, they are essentially close to those observed in the initial tunicate mantle (Van Daele et al. 1992). Therefore, neither the hydrolytic treatment, nor the surface oxidation seems to have any thinning effect on the tunicin whiskers, in contrast with other solid-state treatments such as

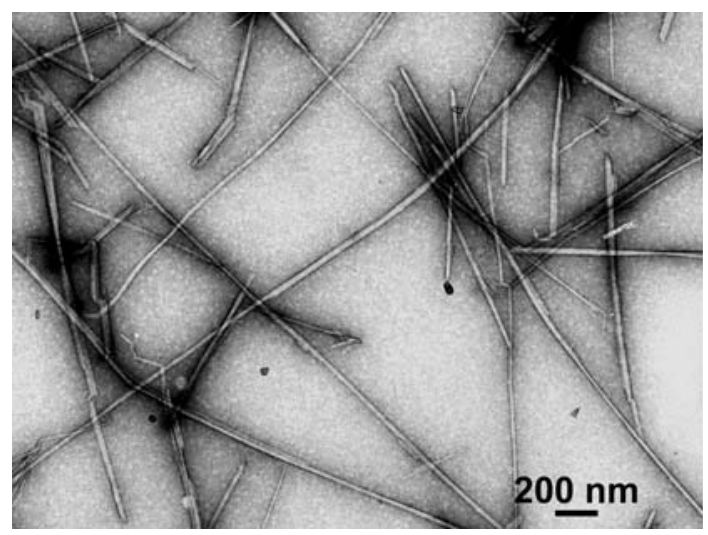

Figure 4. Transmission electron micrograph of the cellulose whiskers after TEMPO-mediated oxidation (molar ratio of $\mathrm{NaOCl} / \mathrm{AGU}=0.5$ eq.). 
acetylation (Sassi and Chanzy 1995) or enzymatic digestion (Chanzy and Henrissat 1983).

In Figure 4, the individual oxidized whiskers are well separated from one another, in contrast with the situation in the initial sample after only $\mathrm{HCl}$ hydrolysis (picture not shown), where the whiskers were clumped together in large aggregates. In fact, the dispersion of the whiskers in Figure 4 is the same as the one in suspensions of tunicin whiskers prepared with the sulfuric acid method (Favier et al. 1995; Sassi and Chanzy 1995; Azizi Samir et al. 2005). In these, the homogeneous dispersion results from the electrostatic repulsion created by the surface sulfate groups. In the case of cotton whisker preparations it has been estimated that about $10 \%$ of the surface hydroxymethyl groups of the AGU were sulfated (Fleming et al. 2001). In the present work, homogeneous suspensions were obtained at DO as low as 0.06 . As in the case of surface sulfate groups, this value is reached when about $10 \%$ of the surface hydroxyl groups are carboxylated (Table 1).

The comparison of the diffraction diagrams of the whiskers before and after surface oxidation indicates that their crystallinity is not affected by the oxidation treatment. Figure 5 illustrates this point as the two powder spectra of the initial (Figure 5A) and oxidized samples (Figure 5B) are nearly identical, both in the sharpness and intensity of the diffraction rings (Figure 5C, D). The two spectra consist of a number of sharp diffraction rings of various intensities. Among these the most prominent are calibrated with d-spacings at $0.258 \mathrm{~nm}$ (medium), $0.39 \mathrm{~nm}$ (very strong), $0.43 \mathrm{~nm}$ (medium), $0.54 \mathrm{~nm}$ (strong) and $0.60 \mathrm{~nm}$ (strong), thus confirming the $\mathrm{I} \beta$ character of this

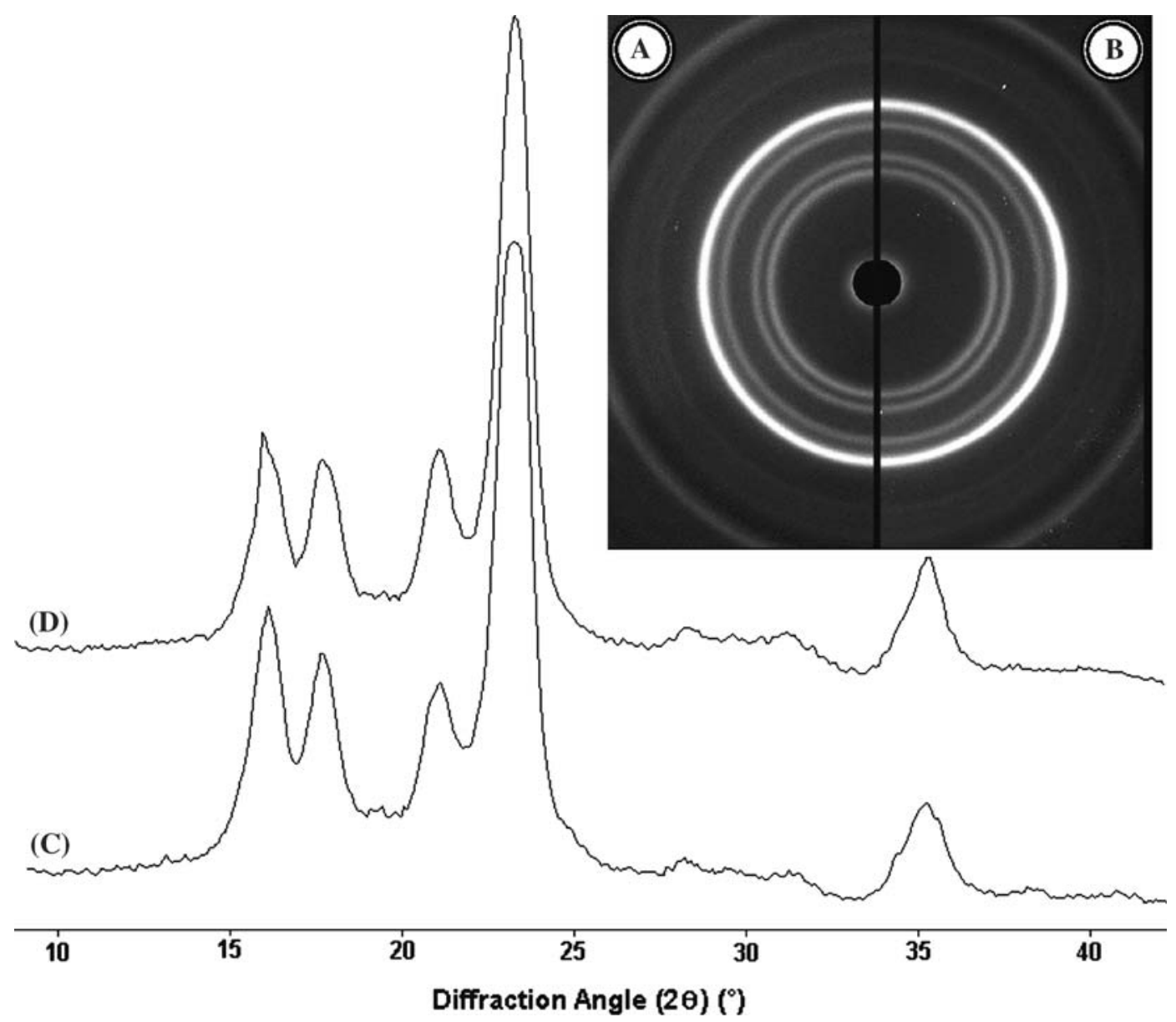

Figure 5. X-ray powder diffraction patterns of cellulose whiskers (A) before and (B) after TEMPO-mediated oxidation (molar ratio of $\mathrm{NaOCl} / \mathrm{AGU}=0.5$ eq.). (C) and (D) correspond respectively to radial traces of the $\mathrm{A}$ and $\mathrm{B}$ diagrams. 
cellulose (Sugiyama et al. 1991; Nishiyama et al. 2002). Within experimental error, the measured crystallinity was of $80 \%$ in both the initial sample as well as in the sample treated with a $\mathrm{NaOCl} /$ AGU ratio of 0.5 eq. This observation confirms that the crystalline core of the whiskers is not affected by the oxidative treatment, which is therefore limited only to the whisker surfaces. This modified surface cannot be detected by diffraction analysis since it gives rise only to a background signal that is essentially the same if the surfaces are hydroxylated or partially carboxylated.

\section{Birefringence of the aqueous whisker suspensions}

One of the interesting properties of the aqueous suspensions of $\mathrm{H}_{2} \mathrm{SO}_{4}$-prepared cellulose whiskers is their ability to self-organize into liquid crystalline arrangement when they are concentrated. In the case of cotton or wood cellulose whiskers, this selforganization is revealed by the observation of fingerprint patterns, indicative of a chiral-nematic ordering when the suspensions are visualized by polarized optical microscopy (Revol et al. 1992). In the present case, the observation of our suspensions between cross polarizers (Figure 6) indicates that the whisker dispersions which were flocculated initially (Figure 6A) became homogeneous and strongly birefringent after surface oxidation (Figure $6 \mathrm{~B}$ ), fulfilling therefore one of the goal of this study. A suspension such as shown in Figure 6B was quite stable as there was no birefringence modification, even after a number of hours and no phase separation could be observed. A close examination of Figure $6 \mathrm{~B}$ indicates that the birefringence is not uniform, but consist rather of domains of various sizes and colors. These observations indicate that the whiskers are organized locally into oriented domains, but the absence of either tactoids (Revol et al. 1992) or fingerprints indicates that the more perfect chiral-nematic order could not be reached. One can speculate for this lack of further organization: it may be due to either a too high polydispersity of the length of the tunicin whiskers or to a too high viscosity of the suspensions if concentrations higher than $0.5 \%$ are used. In fact, other studies dealing with sulfated tunicin whiskers have also shown similar suspensions consisting of birefringent domains (De Souza Lima and Borsali 2002; Azizi Samir et al. 2005), but as in

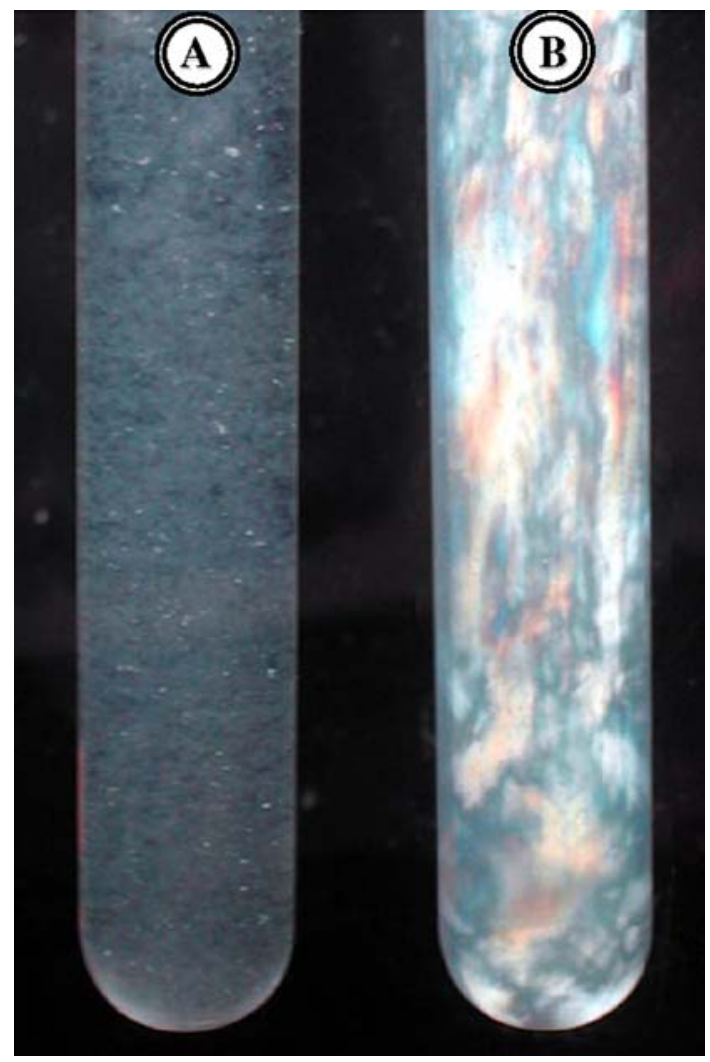

Figure 6. Aqueous $0.53 \%(\mathrm{w} / \mathrm{v})$ suspensions of cellulose whiskers observed between crossed polarizers: (A) before and (B) after TEMPO-mediated oxidation (molar ratio of $\mathrm{NaOCl}$ / $\mathrm{AGU}=0.5)$.

the present case, these suspensions never reached the chiral-nematic order.

\section{Conclusion}

The TEMPO-mediated oxidation of cellulose whiskers resulting from $\mathrm{HCl}$ hydrolysis of tunicin was investigated. By systematically varying the amount of $\mathrm{NaOCl} / \mathrm{AGU}$, conditions were found where all the accessible hydroxymethyl groups located at the whiskers surface were carboxylated, but the core of the crystals remained totally unaffected. One aspect of this derivatization is that it creates a number of charges at the whisker surface and as a consequence these whiskers become easily dispersible in water as birefringent non-flocculated suspensions. So far such homogeneous dispersions have been obtained essentially by the addition of rather labile sulfate 
groups. The present conversion of the surface hydroxymethyl groups into carboxyl entities is much more permanent as these groups are stable and not removable. For further applications, these groups, which are amenable to chemical modification by crosslinking open the ways to prepare cellulose whiskers with a spectrum of surface functionalities.

\section{Acknowledgements}

The authors thank J-L. Putaux for the TEM picture. We wish to dedicate this paper to the late Dr. Jean-François Revol who has been the main inspirator in the development and use of the suspensions of cellulose whiskers.

\section{References}

Araki J., Wada M., Kuga S. and Okano T. 1998. Flow properties of microcrystalline cellulose suspensions prepared by acid treatment. Colloid Surf. A. 142: 75-82.

Araki J., Wada M. and Kuga S. 2001. Steric stabilization of cellulose microcrystal suspension by poly(ethylene glycol) grafting. Langmuir 17: 21-27.

Azizi Samir M.A.S., Alloin F. and Dufresne A. 2005. Review of recent research into cellulose whiskers, their properties and their application in nanocomposite field. Biomacromolecules 6: $612-626$.

Battista O.A. 1950. Hydrolysis and crystallization of cellulose. Ind. Eng. Chem. 42: 502-507.

Battista O.A., Coppick S., Howsmon J.A., Morehead F.F. and Sisson W.A. 1956. Level-off degree of polymerization. Relation to polyphase structure of cellulose fibers. Ind. Eng. Chem. 48: 333-335.

Chanzy H. and Henrissat B. 1983. Electron microscopy study of the enzymic hydrolysis of Valonia cellulose. Carbohydr. Polym. 3: $161-173$.

Da Silva Perez D., Montanari S. and Vignon M.R. 2003. TEMPO-mediated oxidation of cellulose III. Biomacromolecules 4: 1417-1425.

de Nooy A.E.J., Besemer A.C. and van Bekkum H. 1994. Highly selective TEMPO mediated oxidation of primary alcohol groups in polysaccharides. Recl. Trav. Chim. PaysBas 113: 165-166.

De Souza Lima M.M. and Borsali R. 2002. Static and dynamic light scattering from polyelectrolyte microcrystal cellulose. Langmuir 18: $992-996$.

De Souza Lima M.M. and Borsali R. 2004. Rodlike cellulose microcrystals: structure, properties and applications. Macromol. Rapid Commun. 25: 771-787.

Dong X.M., Kimura T., Revol J-F. and Gray D.G. 1996. Effect of ionic strength of the isotropic-chiral nematic phase transition of suspensions of cellulose crystallites. Langmuir 12: 2076-2082.
Favier V., Chanzy H. and Cavaillé J-Y. 1995. Polymer nanocomposites reinforced by cellulose whiskers. Macromolecules 28: 6365-6367.

Fleming K., Gray D.G. and Matthews S. 2001. Cellulose crystallites. Chem. Eur. 7: 1831-1835.

Folda T., Hoffman H., Chanzy H. and Smith P. 1988. Liquid crystalline suspensions of poly(tetrafluoroethylene) "whiskers". Nature 333: 55-56.

Helbert W., Nishiyama Y., Okano T. and Sugiyama J. 1998. Molecular imaging of Halocynthia papillosa cellulose. J. Struct. Biol. 124: $42-50$.

Isogai A. and Kato Y. 1998. Preparation of polyuronic acid from cellulose by TEMPO-mediated oxidation. Cellulose 5: $153-164$.

Kai A. 1976. The fine structure of Valonia microfibril. Gel permeation chromatographic studies of Valonia cellulose. Sen-i Gakkaishi 32: T326-T334.

Livolant F. and Leforestier A. 1996. Condensed phases of DNA: structures and phase transitions. Progr. Polym. Sci. 21: 1115-1164.

Montanari S., Roumani M., Heux L. and Vignon M.R. 2005. Topochemistry of carboxylated cellulose nanocrystals resulting from TEMPO-mediated oxidation. Macromolecules 38: $1665-1671$.

Nishiyama Y., Langan P. and Chanzy H. 2002. Crystal structure and hydrogen-bonding system in cellulose I $\beta$ from synchrotron X-ray and neutron fiber diffraction. J. Am. Chem. Soc. 124: $9074-9082$.

Oster G. 1950. Two-phase formation in solutions of tobacco mosaic virus and the problem of long range forces. J. Gen. Physiol. 33: 445-463.

Rånby B.G. 1951. The colloidal properties of cellulose micelles. Discuss. Farad. Soc. 11: 158-164.

Revol J-F., Bradford H., Giasson J., Marchessault R.H. and Gray D.G. 1992. Helicoidal self-ordering of cellulose microfibrils in aqueous suspension. Int. J. Biol. Macromol. 14: $170-172$.

Saito T. and Isogai A. 2004. TEMPO-mediated oxidation of native cellulose. The effect of oxidation conditions on chemical and crystal structures of the water-insoluble fractions. Biomacromolecules 5: 1983-1989.

Saito T., Shibata I., Isogai A., Suguri N. and Sumikawa N. 2005. Distribution of carboxylate groups introduced into cotton linters by the TEMPO-mediated oxidation. Carbohydr. Polym. 61: 414-419.

Saito T., Nishiyama Y., Putaux J.-L., Vignon M. and Isogai A. 2006. Homogeneous suspensions of individualized microfibrils from TEMPO-catalyzed oxidation of native cellulose. Biomacromolecules 7: 1687-1691.

Sassi J.-F. and Chanzy H. 1995. Ultrastructural aspects of the acetylation of cellulose. Cellulose 2: 111-127.

Segal L., Creely J.J., Martin A.E. Jr. and Conrad C.M. 1959. An empirical method for estimating the degree of crystallinity of native cellulose using X-ray diffractometer. Text. Res. J. 29: $786-794$.

Sugiyama J., Vuong R. and Chanzy H. 1991. Electron diffraction study on the two crystalline phases occurring in native cellulose from an algal cell wall. Macromolecules 24: 4168-4175.

Tahiri C. and Vignon M.R. 2000. TEMPO-oxidation of cellulose: synthesis and characterization of polyglucuronans. Cellulose 7: 177-188. 
Terech P., Chazeau L. and Cavaillé J.-Y. 1999. A small angle scattering study of cellulose whiskers in aqueous suspensions. Macromolecules 32: 1872-1875.

Van Daele Y., Revol J.-F., Gaill F. and Goffinet G. 1992. Characterization and supramolecular architecture of the cellulose-protein fibrils in the tunic of the sea peach (Halo- cynthia papillosa, Ascidiacea, Urochordata). Biol. Cell. 76: $87-96$.

Wise L.E., Murphy M. and d'Addieco A.A. 1946. Chlorite holocellulose, its fractionation and bearing on summative wood analysis and on studies of the hemicelluloses. Paper Trade J. 122: 35-43. 